\title{
LEXICOGRAFÍA, TRADUCCIÓN Y TERMINOLOGÍA: RELACIONES A PARTIR DE DELLA GEOMETRIA DI ORONTIO FINEO TRADOTTE DA COSIMO BARTOLI (VENETIA, 1587)*
}

\author{
Francisco Javier Sánchez Martín \\ javisanmar@um.es \\ Universidad de Murcia
}

\begin{abstract}
Resumen
Los libros matemáticos compuestos por el matemático francés Oronce Finé gozaron de una importante difusión en el siglo XVI, como evidencian las traducciones de su obra sobre geometría, óptica, geografía y astronomía: Protomathesis. Opus varium. Nuestro interés, en esta ocasión, se centra en Della Geometria, el segundo de los libros del volumen Opere di Orontio Fineo del Delfinato, divise in cinque parti, traducida por Cosimo Bartoli (Venetia, Francesco Franceschi Senese, 1587). Realizamos un estudio terminológico comparado entre el texto fuente y esta traducción italiana, lo que nos permitirá observar los procesos de convergencias léxicas propios de este léxico de origen grecolatino, por un lado, así como estudiar los neologismos formales y semánticos en este ámbito, por otro. Finalmente, constataremos el empleo de idénticos mecanismos para la difusión de este vocabulario científico, tanto en latín como en italiano.
\end{abstract}

\begin{abstract}
"Lexicography, translation and terminology: relationships derived from Della Geometria di Orontio Fineo tradotte da Cosimo Bartoli (Venetia, 1587)"

The mathematical books composed by the French mathematician Oronce Finé gained a great prestige in the sixteenth century, as evidenced by the translations of his work

\footnotetext{
* Este trabajo se integra en la línea del proyecto de investigación FFI2010-16324/FILO, "El Diccionario de la ciencia y de la técnica del Renacimiento (DICTER): fases finales", financiado por el Ministerio de Ciencia e Innovación.
} 
about Geometry, Optics, Geography and Astronomy, Protomathesis: Opus varium. Now, our interest is focused on Della Geometria, the second book of Opere di Orontio Fineo del Delfinato, divise in cinque parti, translated by Cosimo Bartoli (Venetia, Francesco Franceschi Senese, 1587). We carry out a terminological study to compare both works, the source text and this Italian translation. This analysis allows us to examine the processes of lexical convergence which are characteristic in this lexicon of Greco-Roman origin, on the one hand, and to study the formal and the semantic neologisms in this specific field, on the other. Finally, we will verify the use of the same mechanisms to disseminate this technical vocabulary, both in Latin and Italian.

Palabras clave: Traducción. Terminología. Oronce Finé. Geometría.

Keywords: Translation. Terminology. Oronce Fine. Geometry. 


\section{Justificación}

En el Renacimiento, cuando tiene lugar la constitución de la ciencia moderna, debemos situar la atención hacia las disciplinas científicas, dentro de un marco cultural propicio al "proyecto filológico de restitución del legado clásico" (Chaparro 2008: 13). Por lo que se refiere a la ciencia matemática, este interés por los saberes no se ciñó solamente al programa de recuperación de las obras de los grandes matemáticos griegos, tarea con la que algunos humanistas italianos no se conformaron, sino que realizaron igualmente traducciones a las lenguas vulgares, en consonancia con el desarrollo de los nuevos avances en este campo del saber.

Gutiérrez Rodilla (1998: 59) explicó que "en la enseñanza universitaria la tradición medieval se basaba en tener como referente de base un texto escrito en latín". Efectivamente, aún en el siglo XVI, las universidades españolas continuaban contando con los textos de los matemáticos del siglo XIII, si bien se comenzaban a enseñar los conocimientos sobre geometría euclidiana a partir de traducciones romances. Así pues, la progresiva introducción de las diferentes lenguas nacionales en el ámbito cultural, tanto literario como científicointelectual posibilitaron que la lengua latina dejara "de quedar protegida bajo la defensa de su utilidad para cualquier hombre culto", según Codoñer (2006: 747). Ello explica el interés por textos coetáneos, como las obras del profesor de París Oronce Finé (1494-1555).

Como puso de manifiesto Antonella Romano (2004), los libros de Fineo tuvieron una importante difusión y se convirtieron en un referente ya en los primeros programas de enseñanza de las matemáticas realizados por los jesuitas. De entre su extensa producción, sobresale su Protomathesis: Opus varium, que vio la luz en París en 1532. Es un trabajo considerable sobre matemáticas, cosmografía y astrología, y contiene cuatro libros que publicó también separadamente: De Arithmetica practica, De Geometria, De Cosmographia sive mundi Sphaera y De Solaribus Horologiis. El segundo, De Geometria, no obstante, data de 1530, según consta en su portada.

Por lo que se refiere a los contenidos, el libro dedicado a la disciplina geométrica, De Geometria, según comprobamos en el índice, se subdivide en 
dos libros: el primero contiene un prefacio seguido de 15 capítulos y el segundo, que se reparte en otras tres partes, agrupa un total de 33 capítulos.

En nuestro país, Fineo ejerció una influencia destacada en algunos tratados de geometría posteriores compuestos por matemáticos españoles. Así lo reconocen expresamente autores como el bachiller Juan Pérez de Moya, quien lo cita de modo frecuente (cf. Sánchez Martín 2009: 71), si bien las menciones también son de signo contrario, pues el francés fue criticado por matemáticos contemporáneos, por ejemplo, Pedro Núñez Salaciense en De erratis Orontii Finaei (1546).

En otra investigación reciente (Sánchez Martín, en prensa), presentada en el marco de las primeras jornadas sobre historia de la traducción no literaria organizadas por la Universitat de València, con el fin de comprobar similitudes y diferencias de los contenidos tratados (exposición de los tres principios de la geometría y definición de los elementos básicos de la geometría plana euclidiana), realizamos una comparación entre el segundo libro dedicado a la geometría (De Geometria) incluido en su obra Protomathesis, compuesta por el matemático francés en 1532, y las correspondientes versiones en lengua romance: Los dos libros de la geometría práctica de Oroncio Fineo Delphinate (manuscrito de 1553) y Opere di Orontio Fineo del Delfinato (Venecia, 1587). En esta ocasión, nuestro interés se centrará de manera primordial en la versión italiana. Partiremos del análisis terminológico de los primeros siete capítulos para comprobar y estudiar la introducción en la lengua italiana del vocabulario geométrico de origen grecolatino, así como la aparición de los neologismos formales y semánticos en este campo. Para ello es necesario el cotejo de las fuentes lexicográficas y documentales en esa lengua, para lo que nos serviremos de los siguientes repertorios: los diccionarios de la Accademia della Crusca (1612-1923) y el Vocabolario Etimologico della Lingua Italiana (Pianigiani 1907).

\section{Descripción y estudio terminológico de la versión italiana Della Geometria}

Según puede leerse en la portada, la obra Opere di Orontio Fineo del Delfinato, divise in cinque parti; Arimetica, Geometria, Cosmografia e Oriuoli. Tradotte da Cosimo Bartoli, Gentilhouomo, \& Academico Fiorentino: et gli specchi, Tradotti dal Cavalier Ercole Bottrigaro, Gentilhuomo Bolognese, ${ }^{1}$ impresa en Venecia en 1587, es fruto de los matemáticos Cosimo Bartoli y Ercole Bottrigaro. La

1. Manejamos la edición digitalizada que está accesible en la colección digital hispánica de la Biblioteca Nacional de España. 
traducción del Libro Secondo Della Geometria se la debemos a Cosimo Bartoli, claro exponente del humanismo italiano, quien estuvo al servicio de la iglesia y de Cosme I de Medici durante la mayor parte de su vida ${ }^{2}$ y fue uno de los miembros de la Academia degli Umili -él mismo se autodenomina "Gentilhouomo, \& Academico Fiorentino"-, de cuyos reglamentos fue redactor en 1540. Esta Academia transformada en la Accademia Fiorentina tuvo como objetivo principal de sus programas la lengua de la ciencia, como señala Biffi (2011), ${ }^{3}$ así como la recuperación y la "traduzione di testi scientifici dalle lingue classiche, e non solo, per consentire un più facile accesso alle scienze, svincolandole dal problema della mediazione linguistica" (Biffi 2011: 93). En este contexto, por tanto, se inscribe la amplia actividad traductora relacionada con la literatura científica, entre cuya producción despuntan: Larchitettura di Leonbatista Alberti (1550), Manlio Severino Boetio senatore et già consolo romano, Della consolatione de la filosofia (1551), Del Modo di misurare le distantie, le superficie, i corpi, le piante, le provincie, le prospettive, \& tutte le altre cose terrene (1564), Opuscoli morali di Leon Batista Alberti Gentil'huomo Firentino (1568) y Opere di Orontio Fineo del Delfinato (ápud Flores Pazos 1991: 368, 373 y Biffi 2011: 94, 98).

La traducción de la Geometría de Oroncio Fineo sigue la fuente latina, tanto en la división de los primeros cuatro libros -Della pratica della Arimetica, Della Geometria, Della Cosmografia overo della sfera del mondo y De gli Orivoli et quadranti a sole-, como en la subdivisión en capítulos de estos. Sin embargo, como hemos verificado, se separa mínimamente en cuanto a la configuración del Libro Secondo Della Geometria, que no está subdivido en partes, pero contiene los mismos 33 capítulos que la versión original latina. Y en la incorpación del quinto libro, Trattato dello specchio parabolico, obra de Ercole Bottrigaro. $^{4}$

2. "Come è stato più volte notato, non si può parlare del lessico tecnico di Bartoli senza inserire il discorso nella cornice più generale della politica di Cosimo I, che prevede l'impiego del fiorentino come grimaldello culturale per la promozione del prestigio del neonato Ducato, nel quadro più generale di un suo affrancamento politico dalla diretta signoria spagnola" (Biffi 2011: 93).

3. "Il 23 febbraio 1541 Cosimo I trasforma l'Accademia degli Umidi in Accademia Fiorentina, organo di stato da lui controllato finanche nei programmi. Questi infatti vengono intelligentemente e abilmente incanalati in un settore in via di espansione, lasciato emarginato dalle questioni linguistiche nazionali incentrate soprattutto sulla lingua di cultura alta e in particolar modo letteraria: la lingua della scienza" (Biffi 2011: 93).

4. Una amplia reseña bibliográfica de este humanista italiano puede consultarse en Mischiati (1971). 
Otra particularidad de esta edición en italiano, aunque característica de las obras en Italia, ${ }^{5}$ es que no cuenta con un prólogo del traductor, sino tan solo con la dedicatoria del impresor dirigida a Guidubaldo marchesi dal Monte. Cabe advertir que cerca de la mitad de la producción procedente de las prensas de Francesco Senese son ediciones en vulgar; entre ellas, sobresalen los tratados correspondientes a importantes ramas del conocimiento científicotécnico como la arquitectura, la medicina o las matemáticas. ${ }^{6}$ En sus dedicatorias, que no carecen de los comunes tópicos, el tipógrafo de Siena enfatiza su declarado interés por la difusión de las obras especializadas, como sucede con la traducción objeto de análisis:

Già non hà urtato V. S. Ilustrissima in questo scoglio: ma con pura verità, con bel giudicio, et con vivace ingegno hà saputo applicar l'animo, conoscere, et apprender quelle cose, che tolte di mano al vulgo, sono d'ornamento et di giovamento grande ancora a i gran Signori. Il che tutto, come è stato potente d'operare in me quanto già di sopra ho detto: così hora in questa occasione dell'havere stampato l'opere d'Orontio nella nostra toscana lingua, è stato efficace di fare che io confidi, che una picciola dichiaratione del mio intimo affetto debba esser da lei ricevuta con animo benigno (Fineo 1587: 3r.)

Coincidimos con Sabio y Fernández (1999: 108) en que "el análisis histórico de la traducción, entendido desde una perspectiva descriptiva y sistémica, puede ser un importante instrumento de trabajo para el estudio de la literatura y la historia literaria", además de la historia de la literatura científica y, por ende, de las lenguas implicadas. ${ }^{7}$

Por consiguiente, para acometer el análisis terminológico del libro segundo Della Geometria decidimos incorporar una tabla analítica en la que recopilamos, por un lado, las glosas que presenta el texto latino y las correspondientes traducciones que se anotan en la versión castellana. Por otro, al

5. Como explica Russell (1985: 16), los traductores de este país gozaban de una independencia social y económica de la que carecían otros colegas europeos, por lo que acometían la traducción por iniciativa propia.

6. "Sono numerose le traduzioni sia di classici sia di autori contemporanei. Ricorderemo la Geografia di Strabone, tradotta da Alfonso Bonaccioli, della quale il D. stampò solo la prima parte nel 1562 [...]; le almeno cinque edizioni delle Metamorfosi di Ovidio, tradotte in ottave da Giovanni Andrea dell'Anguillara; la già citata Architettura di L. B. Alberti, nella traduzione di C. Bartoli, il Vitruvio, tradotto da D. Barbaro (anche questo stampato due volte, nel 1567 e nell'84); la Retorica d'Aristotele, tradotta dal concittadino del D., A. Piccolomini (1571) e le Opere di Oronce Finé tradotte ancora dal Bartoli e da Ercole Bottrigari (1587)" (ápud Baldacchini 1988: s.v. De Franceschi, Francesco).

7. "El análisis de la traducción y sus teorías en la Península durante los siglos XIV y XVIII [revela] la importancia del latín, en primer lugar, como lengua de origen, y del griego, del francés, del provenzal y del italiano" (Sabio \& Fernández 1999: 112). 
estar despojada la versión italiana de los equivalentes terminológicos anotados al margen, optamos por recopilar la terminología correspondiente que se incluye bajo los epígrafes de los siete capítulos objeto de examen. Esta tabla analítica nos permite mostrar la terminología geométrica estudiada. Al mismo tiempo, dado que la traducción italiana carece de ellas, podremos dar cuenta de si dichas glosas están integradas en el texto en forma de amplificaciones, técnica corriente adoptada por otros traductores (cf. Russell 1985: 40).

\begin{tabular}{|l|l|l|}
\hline \multicolumn{1}{|c|}{ Finei (1532) } & \multicolumn{1}{|c|}{ Fineo (1553) } & \multicolumn{1}{c|}{ Fineo (1587) } \\
\hline $\begin{array}{l}\text { De ratione principiorum } \\
\text { geometricorum. Caput. I }\end{array}$ & $\begin{array}{l}\text { De la razón de los } \\
\text { principios de Geometría. } \\
\text { Capítulo I }\end{array}$ & $\begin{array}{l}\text { Della ragione de' principii } \\
\text { geometrici. Cap. I }\end{array}$ \\
\hline $\begin{array}{l}\text { Triplex principiorum } \\
\text { geometricum }\end{array}$ & Tres maneras de principios & $\begin{array}{l}\text { La diferenza de' principii è } \\
\text { di tre sorti }\end{array}$ \\
\hline Diffinitio & Difinitión & Diffinitioni \\
\hline Postulatum & Demanda & Domande \\
\hline $\begin{array}{l}\text { Effatum seu communis } \\
\text { sententia }\end{array}$ & Axioma o sententia & Axiomi/ Sententie comuni \\
\hline Hypothesis & Hypóthesi & Concessione \\
\hline Problema & Problema & Problemati \\
\hline Theorema & Theorema & Theoremati \\
\hline $\begin{array}{l}\text { De figura \& eius terminis. } \\
\text { Caput. II }\end{array}$ & $\begin{array}{l}\text { De la figura y sus límites. } \\
\text { Capítulo II }\end{array}$ & $\begin{array}{l}\text { Della figura \& de' suoi } \\
\text { termini. Cap. II }\end{array}$ \\
\hline Terminus & Término & Termini \\
\hline Punctum & Puncto & Punto \\
\hline Recta & Línea recta & Linea diritta \\
\hline Linea obliqua & Obliqua & Linea torta \\
\hline Plana superficies & Superficie plana & Superficie piana \\
\hline Curva & Superficie curva & Superficie curva \\
\hline Solidum & Cuerpo sólido & Corpo solido \\
\hline $\begin{array}{l}\text { De generali figurarum } \\
\text { differentia [...] Caput. III }\end{array}$ & $\begin{array}{l}\text { De la general descriptión de } \\
\text { las figuras [...] Capítulo III }\end{array}$ & $\begin{array}{l}\text { Della general differenza } \\
\text { delle figure [...] Cap. III }\end{array}$ \\
\hline Simplices & $\begin{array}{l}\text { Figuras símplices, llanas y } \\
\text { compuestas }\end{array}$ & Figure piane \& superficiali \\
\hline Figurae planae. Compositae & & $\begin{array}{l}\text { Figure semplici \& } \\
\text { composte. Miste }\end{array}$ \\
\hline Circuli diffinitio & Diffinitión del círculo & Cerchio \\
\hline Diameter circuli & Diámetro del círculo & $\begin{array}{l}\text { Diametro, overo il } \\
\text { dimetiente del cerchio }\end{array}$ \\
\hline Semicirculus & Medio círculo & Mezo cerchio \\
\hline Circunferentiali linea & Línea circular & Linea circonferentiale \\
\hline Circumducitur & Se rebuelve alderredor & Si tira a torno o si gira \\
\hline
\end{tabular}




\begin{tabular}{|c|c|c|}
\hline Maior Sectio & Pedaço mayor del círculo & $\begin{array}{l}\text { Porzione maggiore del mezo } \\
\text { cerchio. Hapsis }\end{array}$ \\
\hline Minor Sectio & Pedaço menor del círculo & $\begin{array}{l}\text { Segamento, o porzione del } \\
\text { cerchio }\end{array}$ \\
\hline Chorda circuli & Cuerda de círculo & Corda \\
\hline Arcus & Arco & Arco \\
\hline $\begin{array}{l}\text { De Angulis, tam planis } \\
\text { quam etiam solidis Caput. } \\
\text { IIII }\end{array}$ & $\begin{array}{l}\text { De los ángulos, ansí llanos } \\
\text { como sólidos. Capítulo IV }\end{array}$ & $\begin{array}{l}\text { Delli angoli, così piani, } \\
\text { come solidi. Cap. IIII }\end{array}$ \\
\hline Quid angulus & Diffinitión del ángulo & Angolo \\
\hline Angulus planus & Ángulo llano & Angolo piano \\
\hline Angulus rectilinaeus & Ángulo rectilíneo & Angolo di linee diritte \\
\hline Curvilinaeus & Ángulo circuvilíneo & Angolo curvilineo \\
\hline Mixtus & Ángulo mezclado & Angolo misto \\
\hline Angulus & Ángulo recto & Angolo retto \\
\hline Acutus angulus & Ángulo agudo & Angolo acuto \\
\hline Obtusus & Obtuso ángulo & $\begin{array}{l}\text { Angolo ottuso (angolo } \\
\text { obliquo) }\end{array}$ \\
\hline $\begin{array}{l}\text { Angulus curvilinaeus } \\
\text { planus }\end{array}$ & Ángulo curvilíneo pláneo & Angolo curvilineo \\
\hline Angulus sphaeralis & Ángulo sphérico & Angoli sferali \\
\hline Angulus contingentiae & Ángulo contingente & $\begin{array}{l}\text { Angolo della contingenza o } \\
\text { del toccamento }\end{array}$ \\
\hline $\begin{array}{l}\text { Angulus sectionis, eiusque } \\
\text { diversitas }\end{array}$ & $\begin{array}{l}\text { Ángulo de sectión y su } \\
\text { diversidad }\end{array}$ & Angolo della intersegazione \\
\hline Angulus solidus & Ángulo sólido & Angolo solido \\
\hline $\begin{array}{l}\text { Penes quid planorum, } \& \\
\text { rectilinaeorum angulorum } \\
\text { quantitas attendenda Caput. } \\
\text { V }\end{array}$ & $\begin{array}{l}\text { Cómo se ha de consyderar } \\
\text { la quantidad de los ángulos } \\
\text { rectilíneos. Capítulo V }\end{array}$ & $\begin{array}{l}\text { Come si ha da considerare } \\
\text { la quantità delli angoli piani } \\
\& \text { di linee dirette. Cap. V }\end{array}$ \\
\hline $\begin{array}{l}\text { De planis et rectilinaeis } \\
\text { figuris. Caput. VI }\end{array}$ & $\begin{array}{l}\text { De las llanas y rectilíneas } \\
\text { figuras Capítulo VI }\end{array}$ & $\begin{array}{l}\text { Delle figure piane \& di linee } \\
\text { diritte. Cap. VI } \\
\end{array}$ \\
\hline Triangulum & Triángulo & Triangolo \\
\hline Oxygonium & Oxygonio & \begin{tabular}{|l|} 
Triangolo di lati uguali, da \\
Greco detto Oxigonio \\
\end{tabular} \\
\hline Isosceles & Isósceles & $\begin{array}{l}\text { Da Greco detto Isocele, cioè } \\
\text { di duo lati iguali }\end{array}$ \\
\hline Scalenum & Scaleno & \begin{tabular}{|l|} 
Di tre lati disuguali, de \\
Greci detto Scaleno \\
\end{tabular} \\
\hline Quadratum & Quadrado & Quadrato \\
\hline Altera parte longius & Quadrado prolongado. & Quadrilungo \\
\hline Rhombus & $\begin{array}{l}\text { Rhombo (anotado: mas } \\
\text { de los ángulos contrarios } \\
\text { iguales como es }\end{array}$ & Rombo o Mandorla \\
\hline
\end{tabular}




\begin{tabular}{|l|l|l|}
\hline Rhomboides & $\begin{array}{l}\text { (anotado: Quando no } \\
\text { tuviere) Romboides }\end{array}$ & $\begin{array}{l}\text { Romboide, cioè una specie } \\
\text { di mandorla }\end{array}$ \\
\hline Parallelogramum & Paralelogramo & Parallellogramo \\
\hline Trapezium & Trapezias & Trapezie \\
\hline Multilaterae figurae & Figuras multiláteras & $\begin{array}{l}\text { Figure di multi lati ò di } \\
\text { molti angoli }\end{array}$ \\
\hline Gnomon & Qué cosa es el gnomon & Gnomone \\
\hline $\begin{array}{l}\text { De solidis figuris. Caput. } \\
\text { VII }\end{array}$ & $\begin{array}{l}\text { De las figuras sólidas. } \\
\text { Capítulo VII }\end{array}$ & Delle figure solide. Cap. VII \\
\hline Sphaerae diffinitio & Deffinitión de la sphera & Sfera \\
\hline Axis sphaerae & Exe de la sphera & Diametro del mezo cerchio \\
\hline Poli & Polos & Poli della sfera \\
\hline Uniformis Orbis & Orbes conformes & Orbe \\
\hline Disformis & Orbes diformes & Orbe disforme \\
\hline Lenticulare & Cuerpo lenticular & $\begin{array}{l}\text { Corpo grosso come una } \\
\text { lente }\end{array}$ \\
\hline Corpus Ovale & Cuerpo oval & Corpo avato \\
\hline Cubum & Cubo & Cubo o dado \\
\hline Penthagonum & Pentágono & Pentagono \\
\hline Octogonum & Octágono & Ottagono \\
\hline Hexagonum & Hexágono & Exagono \\
\hline Chylindrus & Cylindro & Cylindro \\
\hline Pyramis & Pyrámide & Pyramide \\
\hline & & \\
\hline
\end{tabular}

Efectivamente, puesto que la principal fuente de esta ciencia está en el fondo grecolatino, una primera aproximación a ambos romances denota la correspondencia terminológica. No obstante, un examen detenido revela las divergencias con respecto a la denominación latina en las soluciones propuestas por el traductor italiano. Este aparca el significante latino y prefiere trasladar los contenidos acudiendo bien al fondo patrimonial bien recurriendo a distintos recursos traductológicos, como las paráfrasis, las perífrasis glosadoras y otras reformulaciones. El recurso a estos últimos, amén de otros como las analogías, no siempre implica una carencia terminológica en la lengua meta, como veremos en numerosos ejemplos, de ahí la necesidad de acudir a las fuentes lexicográficas en italiano para dilucidar si, en esos casos, nos hallamos ante soluciones neológicas. ${ }^{8}$

8. Sobre el léxico técnico en las obras de Cosimo Bartoli, Biffi (2011: 97) anotó ya: "Il passo è interessantissimo: Bartoli non solo vi dimostra che i grecismi e i latinismi possono spesso trovare un facile e quotidiano traducente fiorentino, ma mostra anche due dei processi formativi più produttivi del lessico tecnico: l'uso metaforico («aprendosi a 


\subsection{Correspondencias terminológicas}

Las primeras correspondencias terminológicas entre la lengua latina y la romance se observan en las voces que designan los elementos básicos de la geometría, objeto del segundo capítulo "Della figura \& de' suoi termini", según constatamos en términos como figura, termine, punto, linea, superficie, linea diritta, superficie piana y superficie curva, con la salvedad de la designación de la línea oblicua (Obliqua autem linea nuncupatur, en el texto latino). El traductor prefiere acudir a la voz patrimonial torta: "Ma la linea torta è quella che si diffinisce per contraria diffinitione che la diritta, come è quella che le sue parti del mezo non riscontrano a dirittura a i suoi estremi" (Fineo 1587: 2v).

Dicha oposición entre la elección del término culto y el popular la vemos en el pasaje siguiente, por ejemplo, en el uso de cerchio y mezzo cerchio por circulus y semicirculus, respectivamente. Así, en el capítulo tercero se describe el círculo y se enumeran las partes de dicha figura plana. Los correlatos léxicos entre latín e italiano son nuevamente visibles:

Circulus est figura plana superficialis, unica linea (quae circunferentia dicitur) terminata in cuius medio punctum adsignatur, centrum eiusdem circuli nominatum. [...] quod unica circunferentiali claudatur linea (Finei 1532: 51r) / Il cerchio è una figura piana superficiale, terminata da una linea sola, che si chiama la circonferenza, nel mezo della quale si assegna un punto, che si chiama il centro di detto cerchio. [...], che ei sia chiuso da una sola linea circonferentiale ${ }^{9}$ (Fineo 1587: 3r).

Diameter, sive dimetiens circuli nuncupatur (Finei 1532: 51v) / Si chiama il diametro, overo il dimetiente del cerchio (Fineo 1587: 3v).

Semicirculus ergo quem graeci vocant hemicyclium (Finei 1532: 51v) / Il mezzo cerchio adunque, chiamato da Greci Hemiciclo (Fineo 1587: 3v).

Sectio vocat circuli $[\ldots]$ parte circunferentiae semicirculo maiore EFG compraehensa haec à graecis hapsis nuncupatur (Finei 1532: 51v) / Si chiama segamento ò portione del cerchio [...] maggiore [...] come fa la figura EFG, chiamata da i Greci Hapsis (Fineo 1587: 3v).

Chorda circuli/ Corda; Arcus /Arco (Finei 1532: 51v) / (Fineo 1587: 4r)

$\mathrm{Si}$ en el original se ofrecen los dos referentes griego y latino para la misma realidad conceptual, estos suelen respetarse en la traducción: inalterados aparecen

guisa di tromba») o il paragone («un paro di seste da rapportare»); e la possibilità di risemantizzare il lessico comune in chiave tecnica. Due strade, quelle della risementizzazione e dell'uso metaforico, fortemente attestate nella tradizione lessicale delle botteghe artigiane e artistiche, e profondamente radicate in quella linea della tradizione fiorentino-toscana che va da Alberti a Leonardo, fino a Galileo".

9. Circonferentiale no se lematiza hasta el siglo XIX: "Add. Della circonferenze, Appartenente alla circonferenza” (Vocabolario 1863-1923). 


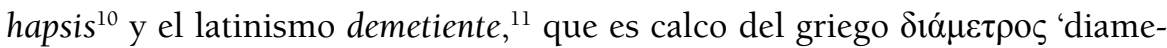
tral'. Más ejemplos los hallamos en el caso de terminología de los triángulos, verbigracia: it. "da Greci detto Oxigonio" por lat. oxigonium.

Tampoco hay apenas cambios en la terminología relativa a la tipología de los ángulos, clasificados según la posición de las rectas y de su abertura:

Planus angulus / Angolo piano

Rectilinaeus porrò angulus / Langolo di linee dirette è quello che si fa di linee diritte

Curvilinaeus autem nominatur angulus / Angolo curvilineo

Mixtus / Langolo misto

Rectus autem angulus / Angolo retto

Acutus angulus / Angolo acuto

Solidus tandem angulus dicit, qui a pluribus duobus planis \& rectilineis angulis, in eodem plano minime constitutis \& ad unum concurrentibus punctum efficit. / [...] \& concorrono ad un punto solo. (Finei 1532: 52r) / (Fineo 1587: 4r-4v).

En la descripción del ángulo sólido, datamos ya el empleo de concorrere en su acepción geométrica que, sin embargo, no aparece lematizada en los diccionarios hasta el siglo XIX: "VII. Detto di linee o di piani, vale Tagliarsi o semplicemente Incontrarsi per effetto del conveniente prolungamento, ed è propriam. Term. de' Geometri" (Vocabolario 1863-1923).

El capítulo sexto, que trata la clasificación y definición de los polígonos, atestigua la recepción de latinismos y helenismos por parte de la lengua italiana, por lo que las correspondencias léxicas son evidentes, como comprobamos en los tecnicismos siguientes: triangulo, oxigonio, isoscele, scaleno, quadrato, quadrangola, parallelogrammo, trapezie, etc.

Finalmente, en el capítulo séptimo tocante a las figuras sólidas no se contemplan grandes diferencias en la presentación de las correlaciones terminológicas (lat. sphaera / it. sfera, diameter / diametro, poli sphaerae / poli della sfera, orbis / orbe, sphaericis /sferiche, pyramis / pyramide, cubum / cubo, chylindrus / cylindro), con la salvedad de la denominación técnica del eje de la esfera y de los significantes que aluden a los cuerpos lenticular y oval, como tendremos ocasión de analizar. No obstante, se sigue de cerca la obra original en el recurso a las analogías -por la forma- empleadas para definir ambos cuerpos:

10. Cf. hap-: "ä $\pi \tau \omega$. II. 9. en gener. estar en contacto. geom. de líneas encontrarse, incidir, Euc. 3 Def. 2" (Adrados 1991).

11. En el texto castellano, el tecnicismo aparece sustituido por la paráfrasis "línea derecha que passa por el centro" (Fineo 1553: 16). 
A maiori quidem sectione corpus lenticulare, ad lentis similitudine crassum Corpus oblongum, instat ovi solidum, ob id ovale nominatum (Finei 1532: $54 r)$.

Dalla portione maggiore cioè, si descrive un corpo grosso come una lente Corpo solido bislongo, come uno uovo, pero si chiama ovato (Fineo 1587: $8 \mathrm{r})$.

\subsection{Divergencias en la traducción: otras posibilidades terminológicas}

Puesto que las soluciones romances propuestas suelen desviarse de la denominación latina existente, en la siguiente tabla reproducimos las divergencias constatadas con respecto al texto de partida. Proporcionamos, igualmente, la primera datación lexicográfica de dichas soluciones en los vocabularios de la Accademia della Crusca.

\begin{tabular}{|c|c|c|}
\hline Finei (1532) & Vocabolario degli Accademici & Fineo $(1587)$ \\
\hline Postulatum & $\begin{array}{l}\text { Dimandagione, e Domandagione. Domanda. Lat. } \\
\text { petitio, postulatio. (Vocabolario 1612) }\end{array}$ & Domande \\
\hline Hypothesis & $\begin{array}{l}\text { Concessione. Concedimento. Lat. concessio } \\
\text { (Vocabolario 1612). }\end{array}$ & Concessione \\
\hline Linea obliqua & $\begin{array}{l}\text { Torto. Avverbio. tortamente. Lat. obliquè } \\
\text { (Vocabolario 1612) } \\
\text { Obbliquo. Non retto, Torto. Lat. obliquus } \\
\text { (Vocabolario 1691) }\end{array}$ & Linea torta \\
\hline Circumducitur & 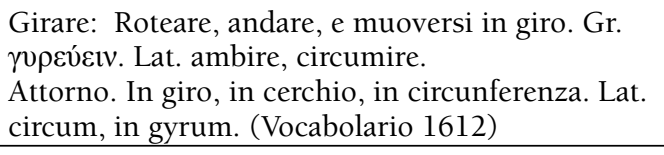 & $\begin{array}{l}\text { Si tira a torno o } \\
\text { si gira }\end{array}$ \\
\hline Diameter circuli & No & Dimetiente \\
\hline Semicirculus & 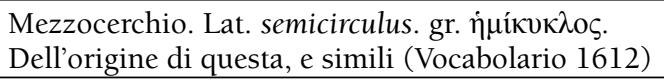 & Mezo cerchio \\
\hline $\begin{array}{l}\text { Minor sectio } \\
\text { circuli }\end{array}$ & $\begin{array}{l}\text { Segamento. Il segare. Lat. sectio (Vocabolario } \\
\text { 1612) } \\
\text { Porzione. Parte. Lat. portio. (Vocabolario 1612) }\end{array}$ & $\begin{array}{l}\text { Segamento, o } \\
\text { porzione del } \\
\text { cerchio }\end{array}$ \\
\hline $\begin{array}{l}\text { Angulus } \\
\text { rectilinaeus }\end{array}$ & $\begin{array}{l}\text { Rettilineo. Figura compresa di linee rette. } \\
\text { (Vocabolario 1691) }\end{array}$ & $\begin{array}{l}\text { Angolo di linee } \\
\text { diritte }\end{array}$ \\
\hline Obtusus & $\begin{array}{l}\text { Obbliquo. Termine matematico, ed è l'opposto a } \\
\text { retto. Lat. obliquus (Vocabolario 1612) }\end{array}$ & $\begin{array}{l}\text { Lottuso (angolo } \\
\text { obliquo) }\end{array}$ \\
\hline Longitudine & $\begin{array}{l}\text { Prima spezie di dimensione, considerata in cosa } \\
\text { materiale. Lat. Longitudo (Vocabolario 1612). }\end{array}$ & Lunghezza \\
\hline Latitudine & $\begin{array}{l}\text { Seconda spezie di dimensione. Lat. Latitudo } \\
\text { (Vocabolario 1612) }\end{array}$ & Larghezza \\
\hline Crassitie & $\begin{array}{l}\text { Astratto di grosso. Lat. crassities, crassitudo } \\
\text { (Vocabolario 1612) }\end{array}$ & Grossezza \\
\hline
\end{tabular}




\begin{tabular}{|c|c|c|}
\hline $\begin{array}{l}\text { Angulus } \\
\text { contingentiae }\end{array}$ & 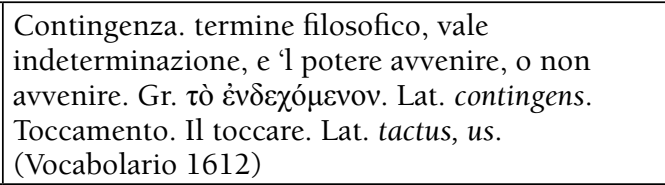 & $\begin{array}{l}\text { Angolo della } \\
\text { contingenza o } \\
\text { del toccamento }\end{array}$ \\
\hline $\begin{array}{l}\text { Angulus } \\
\text { sectionis }\end{array}$ & $\begin{array}{l}\text { Intersecazione. Lo 'ntersecare. Lat. intersecatio, } \\
\text { dicon gli Astrologi. (Vocabolario 1612) }\end{array}$ & $\begin{array}{l}\text { Angolo della } \\
\text { intersegazione }\end{array}$ \\
\hline Oxygonium & No & $\begin{array}{l}\text { Triangolo di lati } \\
\text { uguali, da Greco } \\
\text { detto Oxigonio }\end{array}$ \\
\hline Isosceles & $\begin{array}{l}\text { Isoscele. Dicono i Geometri quel Triangolo, che } \\
\text { abbia due lati uguali. Latin. isosceles, aequicrus. } \\
\text { (Vocabolario 1691) }\end{array}$ & $\begin{array}{l}\text { Da Greco detto } \\
\text { Isocele, cioè di } \\
\text { duo lati iguali }\end{array}$ \\
\hline Scalenum & $\begin{array}{l}\text { Scaleno. Dicono i Geometri quel Triangolo, i lati } \\
\text { del quale son disuguali tra loro. Lat. scalenus. Gr. } \\
\sigma \kappa \alpha \lambda \eta v o ́ \text { s. (Vocabolario 1691) }\end{array}$ & $\begin{array}{l}\text { Di tre lati } \\
\text { disuguali, de } \\
\text { Greci detto } \\
\text { Scaleno }\end{array}$ \\
\hline $\begin{array}{l}\text { Altera parte } \\
\text { longius }\end{array}$ & $\begin{array}{l}\text { Quadrilungo. Figura di quattro lati più lunga, che } \\
\text { larga } \\
\text { (Vocabolario 1729-1738) }\end{array}$ & Quadrilungo \\
\hline Rhombus & $\begin{array}{l}\text { E i matematici chiaman Rombo una figura di } \\
\text { quattro lati, che ha i lati uguali, ma gli angoli } \\
\text { obliqui. Latin. rhombus. (Vocabolario 1612) } \\
\text { Mandorla per similit. alla Figura di Rombo } \\
\text { (Vocabolario 1691) } \\
\text { III. Mandorla, pero similit., vale Figura } \\
\text { romboidale, Rombo, Figura quadrilatera } \\
\text { e equilatera rettilinea, ma non rettangola. } \\
\text { (Vocabolario 1863-1923) }\end{array}$ & $\begin{array}{l}\text { Rombo o } \\
\text { Mandorla }\end{array}$ \\
\hline Rhomboides & $\begin{array}{l}\text { Romboide. Figura prodotta dal rombo. } \\
\text { (Vocabolario 1691) }\end{array}$ & $\begin{array}{l}\text { Romboide, cioè } \\
\text { una specie di } \\
\text { mandorla }\end{array}$ \\
\hline Axis sphaerae & 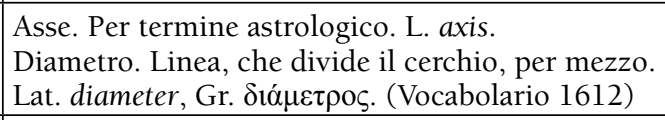 & $\begin{array}{l}\text { Diametro del } \\
\text { mezo cerchio }\end{array}$ \\
\hline Lenticulare & $\begin{array}{l}\text { Lenticolare. Che ha forma e figura simili a } \\
\text { quelle di una lente. Dal basso lat. lenticularis } \\
\text { (Vocabolario 1863-1923) }\end{array}$ & $\begin{array}{l}\text { Corpo grosso } \\
\text { come una lente }\end{array}$ \\
\hline $\begin{array}{l}\text { Corpus } \\
\text { oblongum }\end{array}$ & $\begin{array}{l}\text { Bislungo. Che ha alquanto del lungo, che tende al } \\
\text { lungo: come bistóndo, che ha del tondo, e simili. } \\
\text { Lat. oblongus. (Vocabolario 1612) }\end{array}$ & $\begin{array}{l}\text { Corpo solido } \\
\text { bislongo }\end{array}$ \\
\hline Cubum & $\begin{array}{l}\text { Dado si dice anche a qualunque corpo di sei facce } \\
\text { eguali (Vocabolario 1623) }\end{array}$ & Cubo o dado \\
\hline
\end{tabular}

Ya en el primer capítulo de este Libro Secondo Della Geometria advertimos diferencias entre los textos fuente y meta con respecto a la traducción de los términos que designan los conceptos referidos a los principios de la Geometría: 
Triplicem esse principiorum differentiam, apud omnes, etiam vulgariter eruditos, in confesso est. Dividuntur enim principia, in deffinitiones, postulata $\&$ communes sententias, quas Graecis axiomata, nostri verò effata solent adpellare: quibus suffragantur hypotheses (Finei 1532: 50v).

E gli è chiaro appresso di tutti $\&$ ancora a poco eruditi, che la differenza de principii è di tre sorti: imperoche i principii si dividono in Diffinitioni, Domande \& Sententie comuni; già da Greci chiamati Axiomi \& da Latini Effata, dalle quali sono aiutate le Concessioni (Fineo 1587: 1v).

El traductor italiano conserva los significantes clásicos axioma y effatum 'axioma, predicción', ${ }^{12}$ pero reemplaza el helenismo hypotheses al traducir su significado y opta por la voz derivada concessione (concèdere). En este caso, el préstamo ipotesi en italiano no se encuentra definido hasta la $4^{a}$ edición del Vocabolario della Crusca (1729-1738) "V. G. Supposto. Lat. hypothesis. Gr.

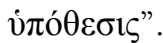

También interviene en la sustitución, al igual que sucede con la versión castellana, del latinismo postulatum: esp. demanda ${ }^{13}$ e it. domande,${ }^{14}$ voz que está lematizada en la primera edición del Vocabolario della Crusca (1612): "Dimanda e Domanda. il dimandare. Lat. interrogatio, petitio". En otro momento Bartoli señala explícitamente la denominación que recibe dicho concepto: "Domande diciamo noi" (Fineo 1587: 2r).

No registramos documentación, en cambio, del tecnicismo postulato en este repertorio, pero sí en el Vocabolario Etimologico de Pianigiani (1907): "Postulato = lat. Postulatum ciò che è domandato, da POSTULARE domandare. Termine di logica. Proposizione semplice, evidente ed assiomatica, che fino dal principio del ragionamento si può chiedere all'avversario di ammettere, senza bisogno di dimostrazione".

Otros conceptos tocantes a los principios de la Geometría son los relativos a los problemas y los teoremas. Las definiciones que ofrece Oroncio Fineo reflejan las dificultades para deslindarlas terminológicamente, inconveniente expresamente declarado por el matemático parisino:

12. "Assioma. Detto comunemente approvato: Massima. Lat. axioma. Gr. $\dot{\alpha} \xi \dot{\xi} \omega \mu \alpha$ " (Vocabolario 1612: s.v.).

13. "Demanda se llama quando el principio no es de todo claro ni fuerça al oyente que lo crea, pero todavía se recibe por principio. Como quien dixiesse: de qualquier punto hasta a qualquier punto se guiará una línea derecha" (Fineo 1553: 13).

14. "Domande diciamo noi che sono quelle che quando una cosa si dice, o si propone, ella è incognita, nè concessa subito da chi l'ode: \& nondimeno, mediante la ragione del principio, ella si comincia ad intendere, \& finalmente si ammette, come è, che da qual si voglia punto si possa tirare una linea ad un'altro punto" (Fineo 1587: 2r). 
Ut non possit ignorari eorundem problematum a theorematis aperta differentia \& mutua singulorum intersese $\&$ problematum $\&$ theorematum subministratio, adeo ut ex antecedentibus omnis subsequentium videatur pendere comprobatio: quatenus rursum ad ipsa deveniatur principia, quemadmodum ex elementorum Euclidis volumine facile manifestatur (Finei 1532: 50v).

El traductor italiano los reproduce sin aclararlos en su traducción: “[...] ch'ei non è possibile il non sapere l'aperta differenza ch'è fra i Problemati \& i Theoremati, che son pure le propositioni" (Fineo 1587: 2r).

Proposizione se define como: "Per detto comunemente approvato, al qual non può contraddirsi, massima, assioma" (Vocabolario 1612). Ya bajo la definición de teorema pueden constatarse las diferencias entre ambos conceptos técnicos: "Teorema. Dimostrazione è prova evidente d'alcuna verità. Lat. theorema. Gr. $\theta \varepsilon \omega ́ \rho \eta \mu \alpha "$ (Vocabolario 1691), que se completa en la cuarta edición para marcar de modo concluyente las diferencias: "Dimostrazione, e Prova evidente d'alcuna verità intorno al supposto, o già fatto, a differenza del Problema, che propone anche il fare" (Vocabolario 1729-1738).

En el segundo capítulo abundan las correspondencias para nombrar los elementos básicos de la geometría, pero, según vimos, el traductor copia torta en lugar de obliqua: "Ma la linea torta è quella che si diffinisce per contraria diffinitione che la diritta, come è quella che le sue parti del mezo non riscontrano a dirittura a i suoi estremi" (Fineo 1587: 2v). Dicha oposición, marcada por la elección entre el término culto y el popular, será una constante de este traductor: así prefiere diritto por retto en todas las ocasiones; o en el uso de cerchio por circulus, al describir esta figura plana.

Detengámonos en la traducción de figura: "La figura è una quantità chiusa da uno, ò pìu termini. Il termine è quello, che è il fine di qual si voglia cosa" (Fineo 1587: 2v). Frente a la traducción española, en la que "fin" o "límite" alternan con término, ${ }^{15}$ el traductor italiano emplea siempre este último, ${ }^{16} \mathrm{a}$ pesar de las correspondencias existentes también en italiano:

15. Son numerosas las concurrencias sinonímicas que hallamos en textos matemáticos renacentistas, alternancias testimoniadas en Nebrija (1495), quien traduce: "Termino por fin: terminus, finis. Termino por linde: limes, finis".

16. Compárense los siguientes pasajes: "Linea igitur, est illatabilis longitudo, latitudine crassitie quem privata: cuius limites sunt puncta, quae etiam a nonnullis signa vocitantur" (Finei 1532: 51r) y "La linea adunque è una lunghezza senza larghezza ò grossezza alcuna, i termini della qualle sono i punti; i quali da alcuni sono ancora chiamati segni" (Fineo 1587: 2v). 
Termine. Parte estrema, stremità, confino. Lat. terminus.

Limite. Termine, confine. Lat. Limes, terminus.

Segno: Per termine. Lat. terminus (Vocabolario 1612)

Observamos igualmente una divergencia en la elección de la expresión que traduce el latín vestigium, cuando se explica el origen matemático de la línea: "Punctum id vocamus [...] Ex cuius intelligibili fluxu, non secus ac si vestigium relinqueret, linea secundum mathematicos causari" (Finei 1532: 51r). Cosimo Bartoli opta por "il segno del suo andare, si dice che si causa la linea secondo i Matematici" (Fineo 1587: 2v), mientras que el matemático Girava traslada: "Los mathemáticos, fingiendo que camina el punto, como si dexasse tras sí algo señalado, dizen que aquel rastro es la línea" (Fineo 1553: 16).

En este mismo capítulo la definición del cuerpo sólido permite ejemplificar bien cómo las formaciones derivadas, disponibles ya en los romances a finales de la Edad Media, son las preferidas frente a los latinismos de dimensión: longitudine (lunghezza), latitudine (larghezza) y crassitie (grossezza); ${ }^{17}$ salvo en profunditate/profundità.

Solidum itaquem dicitur corpus trina dimensione contentum, longitudine videlicet, latitudine, atque crassitie seu profunditate resultans unica superficie (Finei 1532: 51r)

Corpo solido è quello che è contenuto ò composto di tre misure; di lunghezza cioè, e di larghezza \& di grossezza, overo profundità, terminato da una solo ò da piu superficie inmediatamente (Fineo 1587: 3r).

El cultismo crasitud no ha triunfado en la lengua española para denotar 'dimensión' a diferencia de lo que ocurrió en italiano, donde se constata su empleo al menos hasta mediados del siglo XIX: "Crassizie. Termine, e vale Grossezza, grassezza" (Vocabolario 1691), centuria en la que la voz cayó en desuso: "ma è voce oggi pressochè disusata" (Vocabolario 1863-1923).

El capítulo tercero, por su parte, está destinado a la descripción de las figuras geométricas, comenzando por el círculo. A pesar de predominar las coincidencias entre los textos fuente y meta, hay alguna divergencia, principalmente cuando el traductor emplea un desdoblamiento en "segamento ò portione del cerchio" para trasladar "sectio vocant circuli". Dicho procedimiento se utiliza también para hacer accesible el sentido del verbo latino circumduco 'rodear, describir un círculo alrededor', ${ }^{18}$ cuando se describe la construcción matemática del círculo; esto es, "si tira a torno, o si gira uno de

17. "Grossezza. Dimensione che ha un corpo considerato nel suo volume o nella sua circonferenza" (Vocabolario 1863-1923).

18. "Cum in plano recta quaedam linea, extremorum altero intra manente fixo circumducitur" (Finei 1532: 51v). 
suoi estremi" (Fineo 1587: 3v). Esta solución neológica coincide con la ofrecida por Girava, quien emplea el neologismo revolver, que definimos como 'moverse una figura alrededor de un punto o de un eje' (Sánchez Martín 2009: s.v. revolver).

Por lo que respecta a la clasificación tipológica de los ángulos, cabe señalar: primero, la sustitución de rectilineus angulus por la perífrasis "Langolo di linee dirette"; segundo, la constatación -nuevamente- de la preferencia por torto en lugar del latín obliquo al definir el ángulo curvilíneo: "ex obliquarum linearum inclinatione causatur" por "che è causato da linee torte, che vanno a congiungersi insieme" (Fineo 1587: 4r). Más adelante, al retormarse la descripción del ángulo curvilíneo el italiano añade la paráfrasis glosadora "cioè di linee curve", ausente del original latino. Además conviene notar en la reformulación la adición del verbo congiungere o congiugnere ${ }^{19}$ para designar la acción de concurrir o unirse las líneas, y que en la versión castellana de Girava se expresa mediante la voz ayuntar. ${ }^{20}$

La materia tratada en el capítulo sexto concierne a la clasificación y definición de los polígonos:

Triangulum trilaterum / Triangulo de tre lati

Aequilaterum, oxigonium, id est, acutiangulum dicitur / Si chiama triangolo di latti uguali, da Greci detto Oxigonio, cioè dangoli acuti.

Isosceles nominatur / Da Greci detto Isoscele cioè di duo lati uguali.

Sequitur quadrilatera quadrangula. / Segue la di quatro lati quadrangola.

Si autem rectangula sed non aequilatera sit ipsa figura, hoc est, opposita tantum aequalia possidens latera, altera parte longius adpellatur / [...] Si chiama quadrilungo (Finei 1532: 53r) /(Fineo 1587: 6v).

Est enim parallelogrammum, quod sub aequidistantibus lineis continetur / Chiamate da Greci, Parallellograme [...] Imperoche Parallelogramo non vuol dir altro che di linee ugualmente distanti (Finei 1532: 53v) / (Fineo 1587: $7 \mathrm{r})$.

Atestiguamos una preferencia absoluta por las perífrasis glosadoras para explicar los tecnicismos que designan los diferentes polígonos: "de tre lati" por trilaterum, "triangolo di latti uguali" por aequilaterum, "d'angoli acuti" por acutiangulum y "di quatro lati" por quadrilatera.

En otros casos se opta por una reformulación etimológica: en isoscele ("cioè di duo lati uguali") y aequidistantibus ("ugualmente distanti"). ${ }^{21}$

19. "Congiugnere. Mettere insieme, accostare una cosa all'altra. Lat. Coniungere" (Vocabolario 1612).

20. Sobre este término, cf. Sánchez Martín (2009: s.v. ayuntar).

21. "Equidistante. Egualmente distante, e in lat. possiam dire aequè distans, o vero aequaliter distans" (Vocabolario 1612). 
Por el contrario, el circunloquio presente en el original es resuelto mediante la forma neológica, quadrilungo, disponible en esta lengua en el Renacimiento, pese a que su datación lexicográfica se retrasa hasta el siglo XVIII: "Figura di quattro lati più lunga, che larga" (Vocabolario 1729-1738).

En relación a las denominaciones de los cuadriláteros rhombus y rhomboides, destacamos el empleo de la voz mandorla, analogía por la similitud con la forma de óvalo de la almendra, ${ }^{22}$ tanto en la estructura bimembre como en la paráfrasis glosadora que ofrece el traductor italiano: "Di lati uguali ma di angoli disuguali, si suol chiamare Rombo o Mandorla" y "Si suol chiamare una Romboide, cioè una specie di mandorla" (Fineo 1587: 6v).

Es característico del traductor italiano, cuando presenta las denominaciones de los restantes polígonos, la incorporación de una paráfrasis para glosar el significado etimológico de cada tecnicismo:

Multilaterae seu multangulae veniunt adpellandae ... peculiarem denominationem obtinentes. In quarum exemplum habes Penthagonum R, Hexagonum Z, Octogonum Y (Finei 1532: 53v).

Si chiamano figure di molti lati o di molti angoli [...] Per esempio delle quali tu hai il Pentagono cioè, il cinque faccie, R, lo Exagono cioè il sei faccie, Z, \& lo ottagono cioè lo otto faccie (Fineo 1587: 7r).

El último capítulo que examinamos trata de las figuras sólidas. Ya aludimos a que no contemplábamos grandes diferencias en la presentación de las correspondencias terminológicas, salvo en la denominación del 'eje' fuso ${ }^{23}$ por asse ("Per termine matematico. Lat. Axis"), este último ya datado en la primera edición del Vocabolario de la Accademia della Crusca.

Lógicamente, la postura del traductor puede ser dispar. Unas veces no rehúsa dar el término patrimonial ("corpo solido bislongo"); otras, pese a disponer su lengua del significante, puede evitar trasladarlo, como cuando traduce la analogía de forma empleada para definir el cuerpo lenticular. Como podemos leer, ya están en latín estas analogías, procedimiento fructífero en la lengua del Lacio para la creación de numerosa terminología:

A maiori quidem sectione, corpus lenticulare, ad lentis similitudinem crassum quale depingitur per figuram I. A minori porrò circuli sectione, corpus oblongum, instar ovi solidum \& ob id ovale nominatum (Finei 1532: 54r).

Dalla portione maggiore cioè, si descrive un corpo grosso come una lente: come ti dimostra la figura I, $\&$ dalla portinone minore del cerchio, si descrive

22. "Frutta nota. Latin. amygdalum. Grec. $\alpha \mu v ́ \gamma \delta \alpha \lambda o v$. §. Mandorla per similit. alla Figura di Rombo" (Vocabolario 1691).

23. "Et il diametro di esso mezzo cerchio que passa per il centro di esso si acquista nome di fuso" (Fineo 1587: 8v). 
un corpo solido bislongo, come uno uovo, \& però si chiama ovato (Fineo 1587: 8r).

Con idéntica pretensión de hacer accesible el significado emplea los patrimoniales ovato (en "si chiama ovato" por "ovale nominatum") y tondo (por rotundus), formas adjetiva y sustantivada consignadas en la primera edición del diccionario de la Crusca: "Tondo. Di figura rotonda. Lat. rotondus" y "Tondo Sust. figura di forma sferica, circolo, circonferenza. Lat. circulus".

Finalmente la bimembración "cubo o dado" (por "cubum solet adpellari”) refleja una evidente equivalencia semántica.

\section{Conclusión}

La recepción de los tecnicismos matemáticos revisados no plantea problemas a los traductores, como Cosimo Bartoli, decididos a divulgar la ciencia en su idioma, puesto que la nomenclatura científica ya fue acuñada en latín. Así, se vierte en la lengua vulgar la terminología culta clásica, a la vez que se incorporan aquellas palabras comunes que contaban ya en la lengua latina con una semántica especializada. Junto al vocabulario se traducen los mecanismos -analogías, paráfrasis y otras técnicas glosadoras- empleados para trasladar los contenidos ya existentes. En consecuencia, es preciso acudir siempre a la fuente para estudiar la repercusión que esta pudo ejercer en la traducción, así como para dilucidar su papel en la difusión de las innovaciones en la lengua romance receptora.

Además, nuestro traductor tiene la responsabilidad de explicar y hacer accesibles los contenidos geométricos (por ejemplo, definir las figuras sólidas, explicar cómo se genera a partir del punto una línea o describir la construcción matemática de un círculo) que vierte en su lengua, valiéndose de idénticos procedimientos traductológicos (glosas etimológicas, analogías, paráfrasis, etc.). Es entonces cuando surgen las soluciones neológicas, esto es, las divergencias terminológicas con respecto al texto fuente, motivadas por el afán didáctico del divulgador.

\section{Bibliografía}

AcCademia della Crusca. (1612) Vocabolario degli Accademici della Crusca. Edizione I. Venezia: Giovanni Alberti. Versión electrónica: <http://www.lessicografia.it/cruscle/>

ACCADEMia Della CRusca. (1623) Vocabolario degli Accademici della Crusca. Edizione II. Venezia: Iacopo Sarzina. Versión electrónica: <http://www.lessicografia.it/cruscle/> 
AcCademia della Crusca. (1691) Vocabolario degli Accademici della Crusca. Edizione III. Firenze: Stamperia dell' Accademia della Crusca. Versión electrónica: <http://www.lessicografia.it/cruscle/>

ACCAdemia della CRUSCA. (1729-1738) Vocabolario degli Accademici della Crusca. Edizione IV. Firenze: Domenico Maria Manni. Versión electrónica: <http://www.lessicografia.it/cruscle/>

ACCAdemia della CRUSCA. (1863-1923) Vocabolario degli Accademici della Crusca. Edizione V. Firenze: Tipografia Galileiana di M. Cellini. Versión electrónica: <http://www.lessicografia.it/cruscle/>

ADRADOS, Francisco. (1991) Diccionario griego-español. Madrid: CSIC.

BALDACCHINI, Lorenzo. (1988) "De Franceschi, Francesco (Francesco Senese, Senese)." En: Dizionario Biografico degli Italiani. Roma: Istituto dell'Enciclopedia Italiana. Versión electrónica: <http://www.treccani.it/enciclopedia/ francesco-de-franceschi>

BifFI, Marco. (2011) "Il lessico tecnico di Cosimo Bartoli." En: Fiore, Francesco Paolo \& Daniela Lamberini (eds.) 2011. Cosimo Bartoli (1503-1572). Firenze: Olschki, pp. 91-107.

BROCKLISS, Laurence. (1999) "Los planes de estudio." En: Ridder-Symoens, Hilde (ed.) 1999. Historia de la Universidad en Europa. Las universidades en la Europa moderna temprana (1500-1800). Bilbao: Universidad del País Vasco/Euskal Herriko Unibertsitaea, pp. 605-667.

Chaparro Gómez, César. (2008) "La enseñanza de la astronomía en el Renacimiento. El testimonio de Francisco Sánchez de las Brozas." En: Santamaría Hernández, Ma Teresa. (ed.) 2008. La transmisión de la ciencia desde la antigüedad al Renacimiento. Cuenca: Universidad de Castilla-La Mancha, pp. 11-33.

CODOÑER, Carmen. (2006) "Las humanidades en latín." En: Rodríguez, Luis (ed.) 2006. Historia de la Universidad de Salamanca. Salamanca: Ediciones Universidad de Salamanca, pp. 723-756.

FINEI, Orontii. (1532) Protomathesis: Opus varium. Parisiis: Gerardi Morrhii \& Ioannis Petri.

FINEO, Oroncio. (1553) Los dos libros de la geometría práctica de Oroncio Fineo Delphinate, traducidos de latín en lengua española por Pedro Juan de la Estanosa de Bruselas, y dispuestos y ordenados por Hierónimo Girava Tarraconense. Manuscrito. Versión electrónica: < http://dicter.eusal.es/?obra=FineoOroncio >

FInEO, Orontio. (1587) Opere di Orontio Fineo del Delfinato, divise in cinque parti; Arimetica, Geometria, Cosmografia e Oriuoli. Tradotte da Cosimo Bartoli, Gentilhouomo, E Academico Fiorentino: et gli specchi, Tradotti dal Cavalier Ercole Bottrigaro, Gentilhuomo Bolognese. Venetia: Presso Francesco Franceschi Senese.

GutiÉRrez Rodilla, Bertha. (1998) La ciencia empieza en la palabra. Barcelona: Península. 
Mischiati, Oscar. (1971) "Bottrigari, Ercole." En: Dizionario Biografico degli Italiani. Roma: Istituto dell'Enciclopedia Italiana. Versión electrónica: <http:// www.treccani.it/enciclopedia/ercole-bottrigari>

NeBRIJA, Antonio de. (1495) Vocabulario español-latino. Salamanca Ed. facsímil: Madrid: Real Academia Española.

PIANIGIANI, Ottorino. (1907) Vocabolario Etimologico della Lingua Italiana: Versión electrónica: <http://www.etimo.it/?pag=hom>

ROMANO, Antonella. (2004) "El estatuto de las matemáticas hacia 1600." En: Los origenes de la ciencia moderna. Actas Años XI y XII Fundación Orotava de Historia de la Ciencia. Canarias: Consejería de Educación y Cultura, pp. 277-308.

Russell, Peter. (1985) Traducciones y traductores en la Península Ibérica (14001550). Bellaterra: Universidad Autónoma de Barcelona.

SABIO, José Antonio \& $\mathrm{M}^{\mathrm{a}}$ Manuela Fernández. (1999) "La investigación histórica en traducción y la literatura comparada: sobre una antología peninsular de textos teóricos de traducción (siglos XIV-XVIII)." En: Álvarez Sellers, María Rosa (ed.) 1999. Literatura Portuguesa y Literatura Española. Influencias y relaciones. Valencia: Universitat de València, pp. 107-116.

SÁNCHEZ MARTín, Francisco Javier. (2009) Estudio del léxico de la geometría aplicada a la técnica en el Renacimiento hispano. Salamanca: Ediciones Universidad de Salamanca.

SÁNCHEZ MARTín, Francisco Javier. (en prensa) "Las traducciones al español e italiano del libro De Geometria (1532) de Oronce Finé: convergencia terminológica." En: Actas de las I Jornadas sobre historia de la traducción no literaria. Valencia: Universitat de València.

\section{NOTA BIOGRÁFICA /BIONOTE}

Francisco Javier Sánchez Martín es doctor en Filología Hispánica por la Universidad de Salamanca. Ha desarrollado su labor investigadora dentro de diferentes proyectos en el Centro de Investigaciones Lingüística de la Universidad de Salamanca dirigidos por la doctora María Jesús Mancho. Es redactor y miembro del equipo de coordinación del Diccionario de la Ciencia y de la Técnica del Renacimiento (DICTER). Actualmente desempeña su actividad docente e investigadora en la Universidad de Murcia como Profesor Contratado Doctor del Departamento de Lengua Española y Lingüística General. Ha colaborado en la gestión de actividades científicas e intervenido en congresos y seminarios; cuenta con múltiples publicaciones, que se circunscriben a sus líneas de trabajo en lexicografía histórica, historia de la lengua española y estudio del léxico de especialidad. 
Francisco Javier Sánchez Martín holds a PhD in Hispanic Philology from the University of Salamanca. He has developed his research within the projects conducted by Professor María Jesús Mancho in The Center of Linguistic Research at the University of Salamanca. He is copy editor and member of the coordination team of the Dictionary of Science and Technology in the Renaissance (DICTER). He currently carries out his teaching and research at the University of Murcia as Professor in the Department of Spanish Language and General Linguistics. He has worked in the management of scientific activities and participated in conferences and seminars. He holds numerous publications, which are connected with his lines of research in historical lexicography, the history of the Spanish language and the study of specialized vocabulary. 